\title{
Normalizing Parkinsonian networks
}

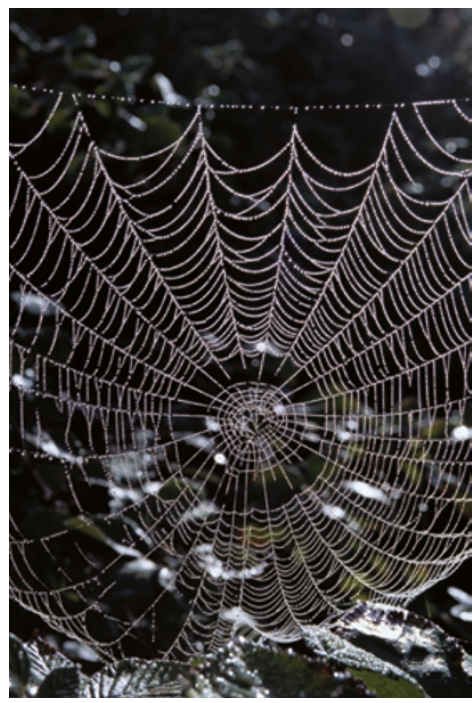

Gene therapy for the treatment of the motor symptoms of Parkinson's disease is in an early stage of development. Earlier this year, Eidelberg and colleagues published the initial results of a therapy involving gene delivery directly to the subthalamic nucleus (STN), which improved the patients' motor function. They now show that this treatment also normalized metabolic changes associated with Parkinson's disease that occur in a network that drives motor activity, providing an objective measure of treatment outcome.
Patients with Parkinson's disease have abnormal metabolic activity - which can be measured using $\left[{ }^{18} \mathrm{~F}\right]$-fluorodeoxyglucose positron emission tomography - both in individual brain regions and in two separate networks, one consisting of regions associated with motor functioning and the other of areas involved in cognition. Here, the authors assessed whether the gene therapy normalized metabolic activity in the patients' brains.

The therapy involved unilateral infusion into the STN of a viral vector containing the gene for glutamic acid decarboxylase (GAD), which catalyzes the decarboxylation of glutamate to GABA ( $\gamma$-aminobutyric acid) and thus reduces glutamatergic neurotransmission in the basal ganglia. The authors measured glucose metabolism in the patients' brains at baseline and 6 and 12 months after surgery.

They showed that the gene therapy altered regional glucose metabolism in the operated hemisphere only, with reductions in the thalamus but increases in the primary motor area and the premotor cortex at both time points.

Network activity was also changed by the gene therapy: the elevation in activity of the motor-associated network that is associated with Parkinson's disease was reduced in the treated hemisphere but not in the untreated hemisphere at 6 and 12 months after gene therapy. Importantly, the decline in network activity correlated with improvement in motor functioning. By contrast, the treatment had no effect on the elevated activity of the cognition-associated network in either hemisphere.

These findings indicate that GAD gene therapy affects both regional and network metabolic activity in patients with Parkinson's disease, in brain areas that are associated with motor function but not in areas involved in cognition. Moreover, they suggest that measurements of activity in metabolic networks can be used to objectively assess the effectiveness of potential new treatments for Parkinson's disease.

Leonie Welberg

ORIGINAL RESEARCH PAPERS Feigin, $\mathrm{A}$, et al. Modulation of metabolic brain networks after subthalamic gene therapy for Parkinson's disease. Proc. Natl Acad. Sci. USA 104, 19559-19564 (2007) | Kaplitt, M. et al. Safety and tolerability of gene therapy with an adeno-associated virus (AVV) borne GAD gene for Parkinson's disease: an open label, phase I trial. Lancet 369, 2097-2105 (2007) 